\title{
COMPORTAMENTO MECÂNICO DO COMPÓSITO PRFV QUANDO IMERSO EM ÁGUA DO MAR POR TEMPO PROLONGADO
}

\author{
R. A. D. CUNHA ${ }^{* 1}$, J. K. D. SANTOS ${ }^{1}$, R. C. T. S. FELIPE ${ }^{2}$ e R. N. B. FELIPE ${ }^{2}$ \\ ${ }^{1}$ Universidade Federal do Rio Grande do Norte - UFRN, Natal - RN, ricardoalexc@gmail.com \\ ${ }^{2}$ Instituto Federal do Rio Grande do Norte - IFRN, Campus Central, Natal - RN \\ ricardoalexc@gmail.com
}

Submetido 03/11/2016 - Aceito 23/06/2017

DOI: $10.15628 /$ holos.2017.5320

\section{RESUMO}

No setor industrial, materiais que apresentem bom desempenho mecânico atrelado com uma vida útil elevada e a uma boa relação custo/benefício vem a cada dia sendo mais procurado, diante disso, os materiais compósitos, dentre eles os compósitos poliméricos fibrosos estão a cada dia sendo mais utilizados, pois estes desempenham determinadas funções que os materiais convencionais não conseguem desempenhar. No entanto, em se tratando dos compósitos poliméricos é importante que se saiba de fato qual o comportamento que este material tem quando está em situações adversas como em contanto com os fluidos de processo ou até mesmo com calor. Desta forma, o objetivo dessa pesquisa foi desenvolver um compósito utilizando uma matriz polimérica de resina poliéster com reforço fibroso de manta de vidro-E, onde este foi imerso em água do mar por um período de 1460 dias. Os corpos de prova (CP's) foram submetidos ao ensaio de tração uniaxial, buscando determinar resistência e módulo de tração. Feito isso, realizou-se um comparativo das propriedades mecânicas para todas as condições de ensaio como estado seco e água do mar. Além disso, foi caracterizada a fratura final dos CP's para a condição de imersão. Ao término dos ensaios observou-se perda nas propriedades mecânicas para a condição de ensaio de tração após o período de imersão, porém esta perda não invalida o seu uso uma vez considerando estes parâmetros durante a elaboração do projeto estrutural.

PALAVRAS-CHAVE: Compósitos Poliméricos; Degradação Mecânica; Água do Mar; Propriedades Mecânicas.

\section{Analysis of Mechanical Properties of PRFV Composite when Immersed in Seawater for Extended Time.}

\section{ABSTRACT}

In the industrial sector, materials that present good mechanical performance linked to a high useful life and at a good cost / benefit comes every day being more sought, in front of this, the composite materials, among them the fibrous polymeric composites are every day being more requested, because they perform certain functions that conventional materials cannot perform. However, when it comes to polymer composites, it is important to know the behavior of this material when it is in adverse situations, such as in process fluids or even heat. In this way, the objective of this research was to develop a composite using a polymer matrix of polyester resin with fibrous reinforcement of E-glass, where it was immersed in sea water for a period of 1460 days. The specimens (CPs) were submitted to the uniaxial tensile test, seeking to determine strength and tensile modulus. After this, a comparative of mechanical properties was performed for all the test conditions as dry state and sea water. In addition, the final fracture of the CPs was characterized for the immersion condition. At the end of the tests, loss of mechanical properties was noticed for the tensile test condition after the immersion period, but this loss does not invalidate its use once considering these parameters during the structural project elaboration.

KEYWORDS: Polymeric Composites, Mechanical Degradation, Seawater, Mechanical Properties. 


\section{INTRODUÇÃO}

Materiais compósitos é definido por Mendonça (2005), como um sistema combinado por dois ou mais materiais a nível macroscópico que trabalham simultaneamente, funcionado como uma unidade visando obter um conjunto de propriedades que nenhum dos componentes individualmente apresentaria. Dentre eles, os compósitos poliméricos reforçados por fibras estão sendo bem utilizados em diversas aplicações na engenharia como em pontes, vasos de pressão, fabricação de aeronaves e etc. (Sathishkmar et al. 2014, Felipe, 2012).

Compósito polimérico reforçado com fibras (FRP) onde as fibras podem ser de carbono, aramida e de vidro imersos em uma matriz de epóxi, são amplamente utilizados para diversas aplicações de engenharia, incluindo aeronaves, dispositivos mecânicos, componentes estruturais navais, pás de turbinas eólicas, e infraestruturas civis (tanto pode ser usado em reparos, onde as estruturas estão necessitando de manutenção, bem como para construções novas) (Loyola et al. 2010). Zhai et al. (2014) afirma que o fraco desempenho nos reparos utilizando os materiais convencionais levou a aplicação de polímero reforçado com fibra (PRF) para serem utilizados em reparos de estruturas de concreto armado; já sendo utilizado para reparos em rodovias, onde a incidência de corrosão é bem acentuada e por ter uma boa relação custo/benefício.

Leonard et al. (2009), destaca o uso dos compósitos poliméricos na indústria aeronáutica, usados na fuselagem e portas do trem de pouso, devido a baixa densidade (leveza) e redução à fadiga nos elementos de fixação utilizados nesses materiais. Porém, seu alto custo é um fator limitante na sua utilização para aplicações comercial, para tanto, pesquisas têm buscado melhorar as propriedades de compósitos e assim, reduzir o custo da preparação e do produto.

O comportamento mecânico de um compósito reforçado com fibras depende basicamente da resistência e módulo de elasticidade das fibras, estabilidade química, resistência da matriz e da interface fibra/matriz para permitir a transferência de carregamento para o sistema estrutural (Gupta et al. 2009). Vários fatores podem ainda influenciar na transferência de carga nos materiais poliméricos reforçados por fibras, tais como o volume de fibra, a orientação das fibras, material de matriz, temperatura, umidade além da espessura das camadas do compósito (Sridhar e Venkatsha 2013).

Apesar das melhorias no desempenho oferecido pelos compósitos poliméricos reforçados por fibras, estes materiais são altamente susceptíveis a danos, tais como a delaminação*, fraturas na matriz, fratura interlaminar e desaderência fibra/matriz, todos estes podem provocar uma falha estrutural (Boger et al. 2009, Todo et al. 1998). Além disso, vários fatores podem ainda ser responsáveis por danos nestes materiais como carregamento excessivo, o impacto, a fadiga, as condições ambientais, o teor de vazios além do processo de fabricação que pode não ser o adequado.

Felipe (2012) afirma que, ao se utilizar um compósito sob a ação de condições adversas como umidade, temperatura e radiação ultravioleta, devem-se levar em consideração seus elementos constituintes e sua influência no comportamento mecânico desse material. Essa influência está diretamente relacionada pelos elementos constituintes já que os compósitos podem ser formados por matrizes metálicas, cerâmicas, carbonosas ou poliméricas. 
Estudos mostram que os compósitos, principalmente os poliméricos, absorvem umidade, e esta absorção depende de fatores como temperatura, fração volumétrica das fibras, orientação do reforço e natureza das fibras, área superficial de exposição, difusividade de massa e proteção superficial. O mecanismo de penetração da umidade se dá basicamente mediante o processo de difusão direta de água dentro da matriz e da fibra, porém, por exemplo, no caso das fibras sintéticas, em menor intensidade. Também existem outros mecanismos de absorção de umidade que podem ser a capilaridade que acontece na interface fibra/matriz devido ao fluxo de moléculas de água ao longo da interface e ainda, o transporte de umidade por microfissuras envolvendo o fluxo de estocagem de água nestas microfissuras, tema abordado tanto por Cavalcante et al. (2005) como em Hull e Clyne (1996).

Diante disso, este trabalho visa o estudo da influência do comportamento mecânico em um compósito utilizando uma matriz polimérica de resina poliéster ortoftálica com reforço fibroso de manta de vidro-E, quando imerso em água do mar por um período de 1460 dias (4 anos). Foi escolhido este meio (água do mar) devido a necessidade de se conhecer o comportamento desse compósito, uma vez que o mesmo possa ser utilizado imerso na água do mar, como por exemplo em plataformas de petróleo, onde as tubulações para transporte de água, em determinadas circunstâncias, ficam submersas no mar. Para caracterização mecânica foram realizados ensaios de tração uniaxial, buscando determinar resistência e módulo de tração. Feito isso, realizou-se um comparativo das propriedades mecânicas para todas as condições de ensaio como estado seco e água do mar. Além disso, foram realizadas análises macroscópicas e microscópicas da característica da fratura para as duas situações em questão.

\section{EXPERIMENTAL}

\subsection{Materiais e métodos}

A matriz polimérica utilizada para a confecção da placa do compósito foi a resina poliéster ortoftálica insaturada, L-120/08 pré-acelerada, fabricada pela NOVOCOL, com as seguintes características fornecidas pelo fabricante: densidade entre 1,10 e 1,15 g/cm ${ }^{3}$; viscosidade BROOKFIELD a 25 으, entre 260 e 300 cP. O catalisador usado para o sistema de cura da resina foi a metil-etil-cetona (MEKP), fabricada pela AKZO NOBEL. O reforço usado no compósito foi a manta de fios picados de vidro do tipo $E$, produzida pela OWENS-CORNING com uma gramatura de $450 \mathrm{~g} / \mathrm{m}^{2}$. A fabricação do compósito se deu mediante o processo de laminação manual (hand lay-up), sendo a resina poliéster, catalisada com $1 \%$ de catalisador referente ao volume total da resina, e como reforço quatro camadas de manta de fios picados de vidro-E, obtendo-se, desta maneira, uma placa, à temperatura ambiente de $28^{\circ} \mathrm{C}$. Após a cura do compósito, foram cortados corpos de prova (CP) para caracterização do compósito e determinação das propriedades de tração uniaxial.

\subsection{Ensaios mecânicos}

Os corpos de prova foram confeccionados a partir da fabricação das placas, sendo estes cortados com disco de corte de fio de diamante. Em seguida, foram confeccionados os corpos de prova para realização dos ensaios de densidade (ASTM D792), calcinação (ASTM D3171) e tração uniaxial (ASTM D3039) para caracterizar e determinar as propriedades mecânicas do compósito. 


\subsection{Característica da fratura final}

Ao término dos ensaios mecânicos foi realizada a análise microscópica da fratura final usando um microscópio eletrônico de varredura (MEV), na área da região de fratura, tanto para o estado original (seco) como para a condição do material imerso em água do mar.

\section{RESULTADOS E DISCUSSÃO}

\subsection{Densidade e teste de calcinação}

A Tabela (1) traz o valor médio da densidade do compósito e as frações volumétricas de matriz, reforço e vazios determinados apenas para a situação do material nas condições originais, ou seja, sem condicionamento. Rafiee e Reshadi (2014) observou esse mesmo valor de densidade, em torno de $1,5 \mathrm{~g} / \mathrm{cm}^{3}$, para uma manta de fibra de vidro costurada e usando uma resina poliéster.

Tabela 1: Densidade e frações volumétricas de matriz, reforço e vazios.

\begin{tabular}{c|c|c|c}
\hline $\begin{array}{c}\text { Densidade } \\
\left(\mathrm{g} / \mathrm{cm}^{3}\right)\end{array}$ & Matriz (\%) & Reforço (\%) & Vazios (\%) \\
\hline $1.52 \pm 0.2$ & $72 \pm 2$ & $26 \pm 2$ & $2 \pm 0.1$ \\
\hline
\end{tabular}

\subsection{Ensaio de tração uniaxial}

Na figura 1 é possível observar o comportamento mecânico quando o compósito foi tracionado nas condições original (seco) e imerso em água do mar por 1460 dias (4 anos). Desta forma, para todas as condições observa-se que o material após ensaio apresentou o mesmo comportamento. Inicialmente um comportamento linear até atingir sua tensão máxima. 

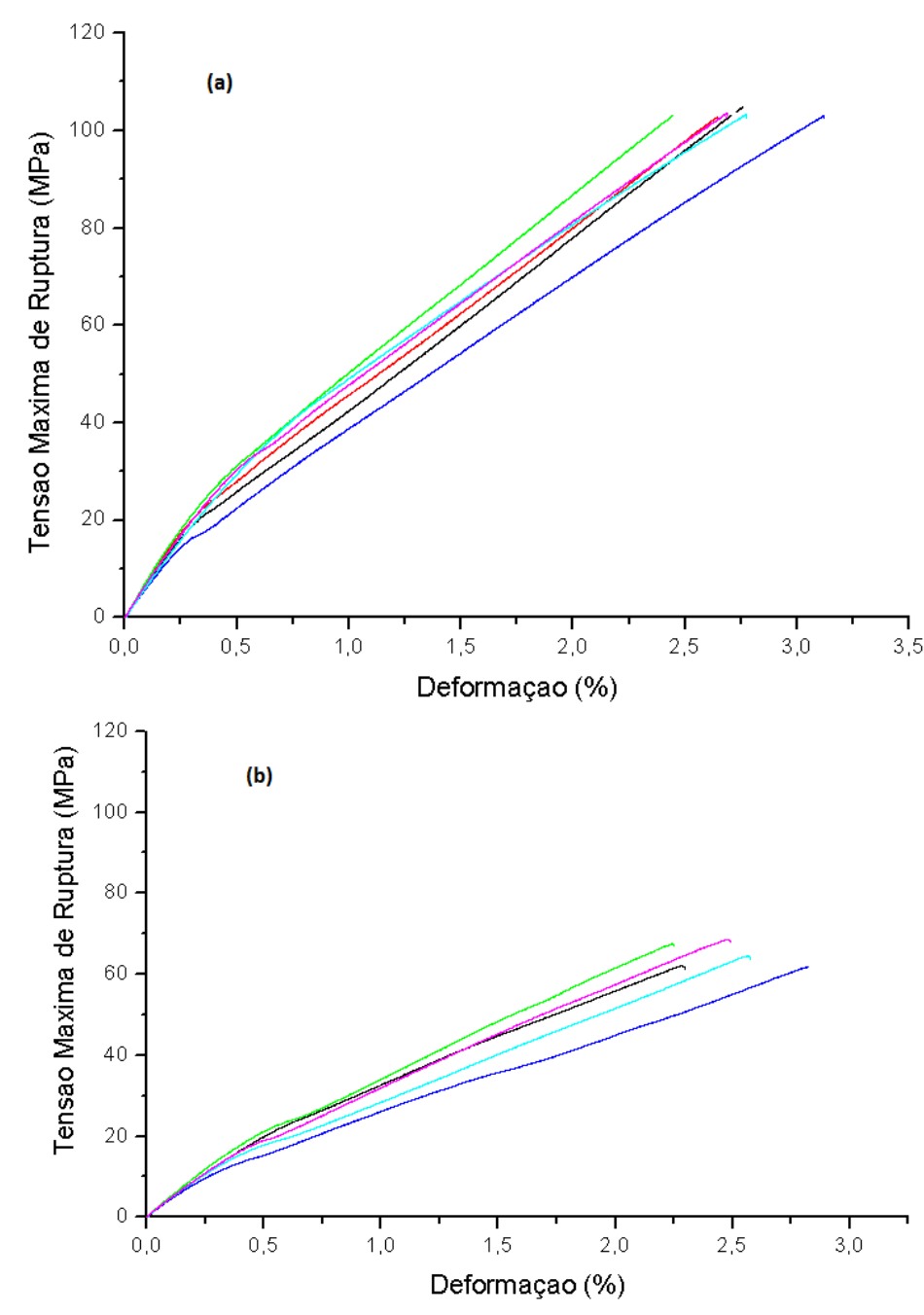

Figura 1: Curva tensão x deformação - Ensaio de tração uniaxial. (a) estado seco (b) imerso em água do mar.

Foi observado, na figura 2, que após condicionamento em água do mar ocorreu uma diminuição da resistência à tração, ou seja, representou um percentual de queda em torno de $30 \%$, podendo essa diminuição ser atribuída ao mecanismo de capilaridade que acontece quando o material é exposto a um ambiente úmido, pois a umidade tende a penetrar por capilaridade e com isso interferir na interface fibra/matriz e consequentemente no comportamento mecânico.

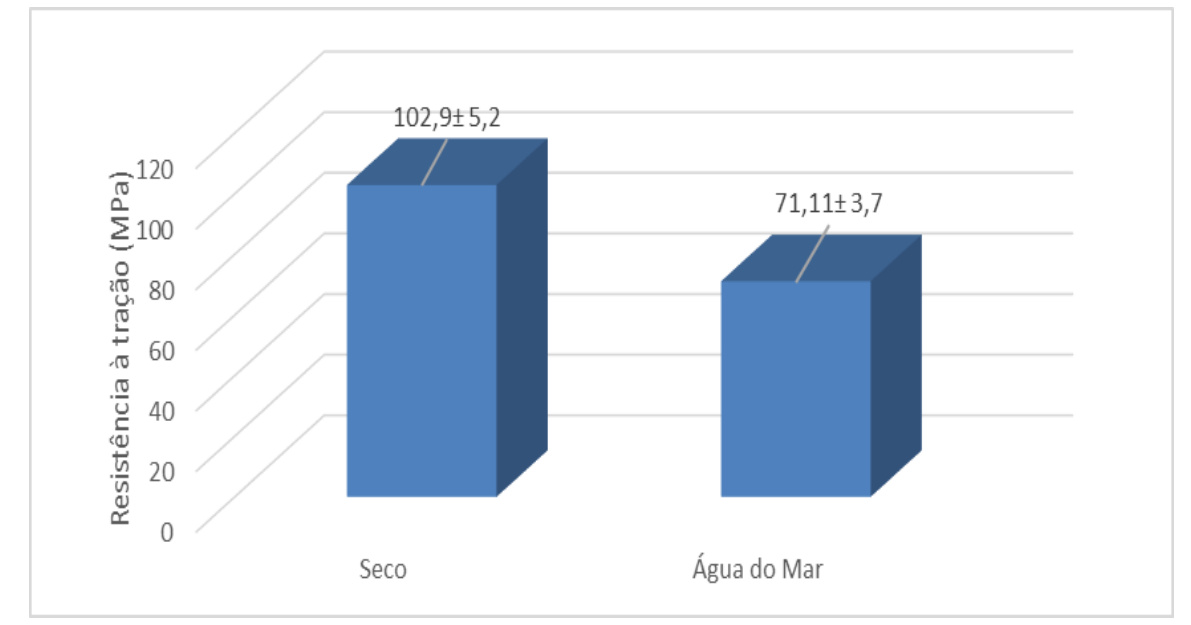

Figura 2: Limite de resistencia à tração dos compósitos nos estados seco e imerso em água do mar. 
No que se refere ao módulo de elasticidade à tração, é possível observar na figura 3, que o compósito no estado seco apresentou maior módulo, com uma diferença de 0,38 GPa para 0,34 GPa, no estado úmido, representando uma queda de $10 \%$, mostrando dessa forma um decréscimo da rigidez do material quando imerso em água, mas se considerarmos o desviopadrão, o valor para o módulo elástico permaneceu constante.

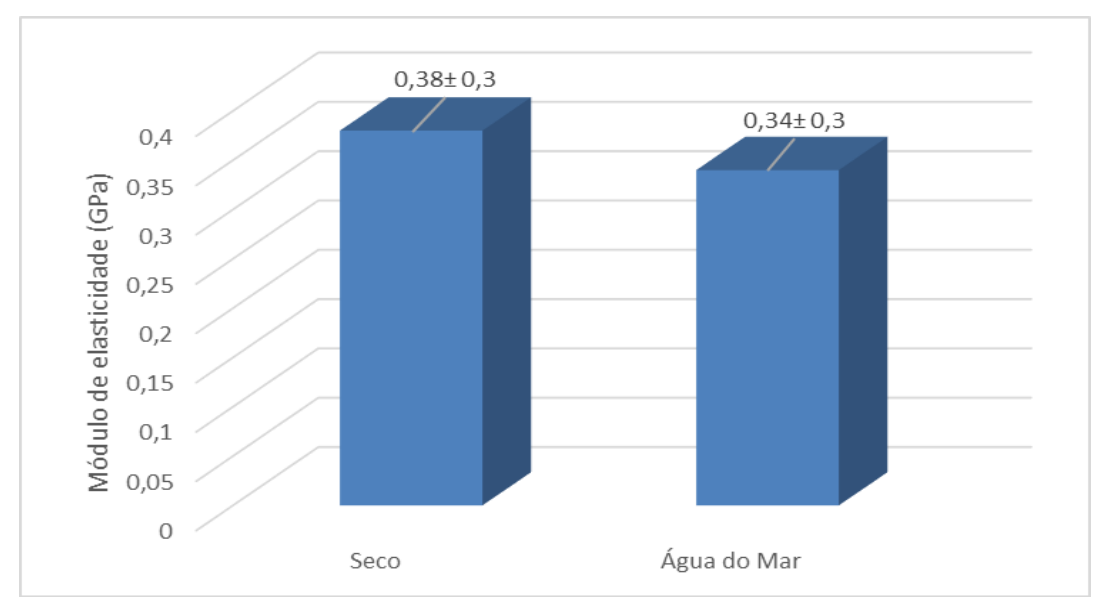

Figura 3: Módulo de elastcidade dos compósitos nos estados seco e imerso em água do mar.

\subsection{Característica da Fratura Final}

A caracterização da fratura final foi feita a partir da análise macroscópica e microscópica, com utilização do MEV, como pode ser observada na figura 4, para o corpo de prova no estado original (seco) e na figura 5 para o corpo de prova após envelhecimento (imerso em água do mar por 4 anos). 

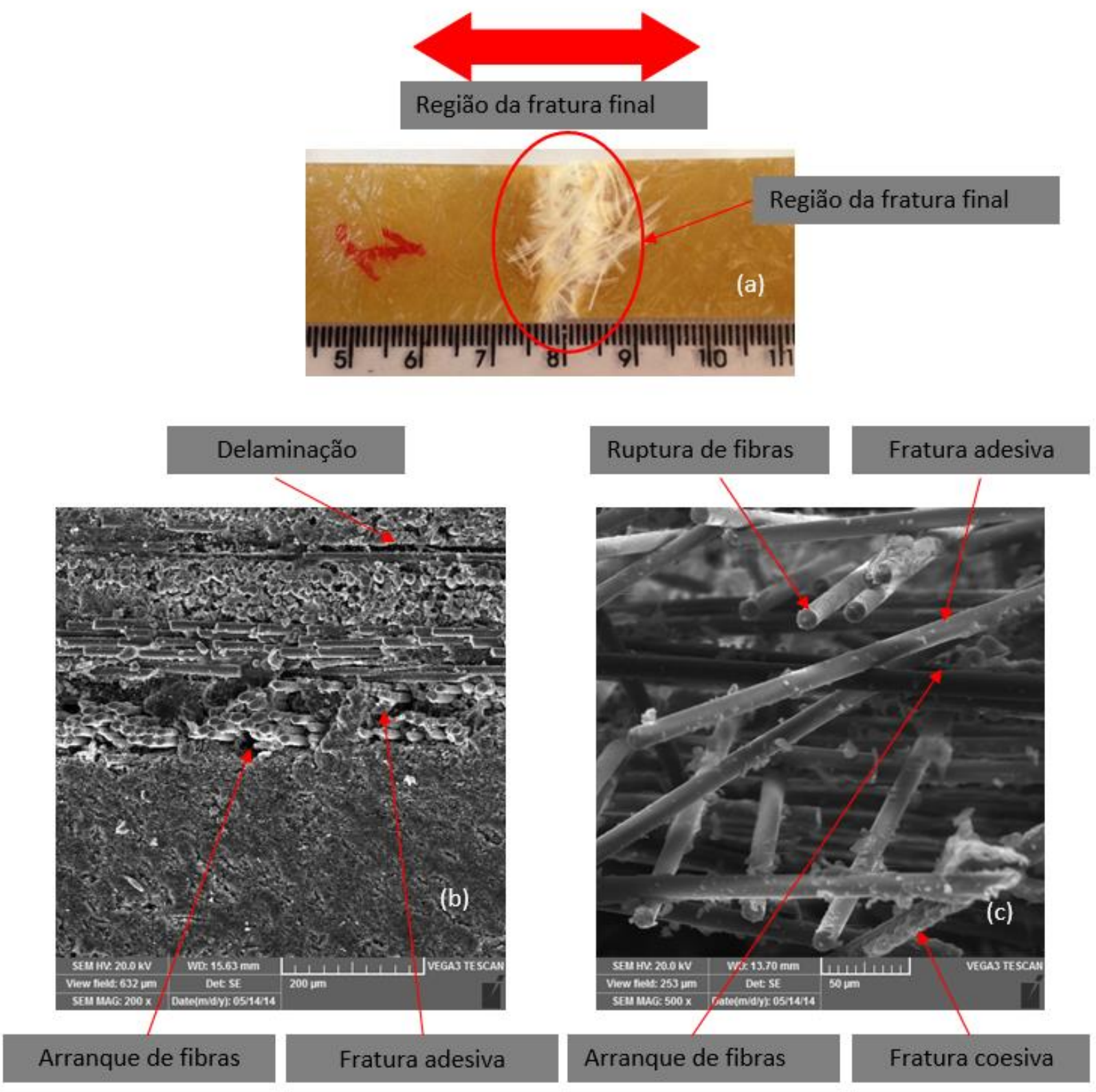

Figura 4: Região da fratura final do corpo de prova tracionado na condição original (estado seco).

Analisando a figura 4(a) é possível observar uma região embranquecida na região da fratura onde se evidenciam a presença de fibras soltas, sendo essa imagem capturada mediante câmara fotográfica digital, apresentando uma característica da desaderência fibra/matriz. Um outro aspecto a ser abordado se refere à característica da fratura final, pois esta aconteceu perpendicular ao esforço de tração, reforçando a definição que o corpo foi realmente rompido pelo esforço de tração. Na figura 4(b), na seção lateral do corpo de prova, verifica-se a presença de delaminação, que segundo a norma ASTM D 2563-08, é definida como a separação entre as camadas internas do compósito, sendo esta evidenciada pela presença de uma fenda . É possível perceber também, arranque de fibras e a fratura adesiva na matriz. Na figura 4(c), referente à seção transversal da fratura final observa-se ruptura e arranque de fibras, e, além disso fratura coesiva na matriz, e ainda fratura adesiva na interface fibra/matriz, que é evidenciada pelo aspecto limpo da superfície das fibras. 

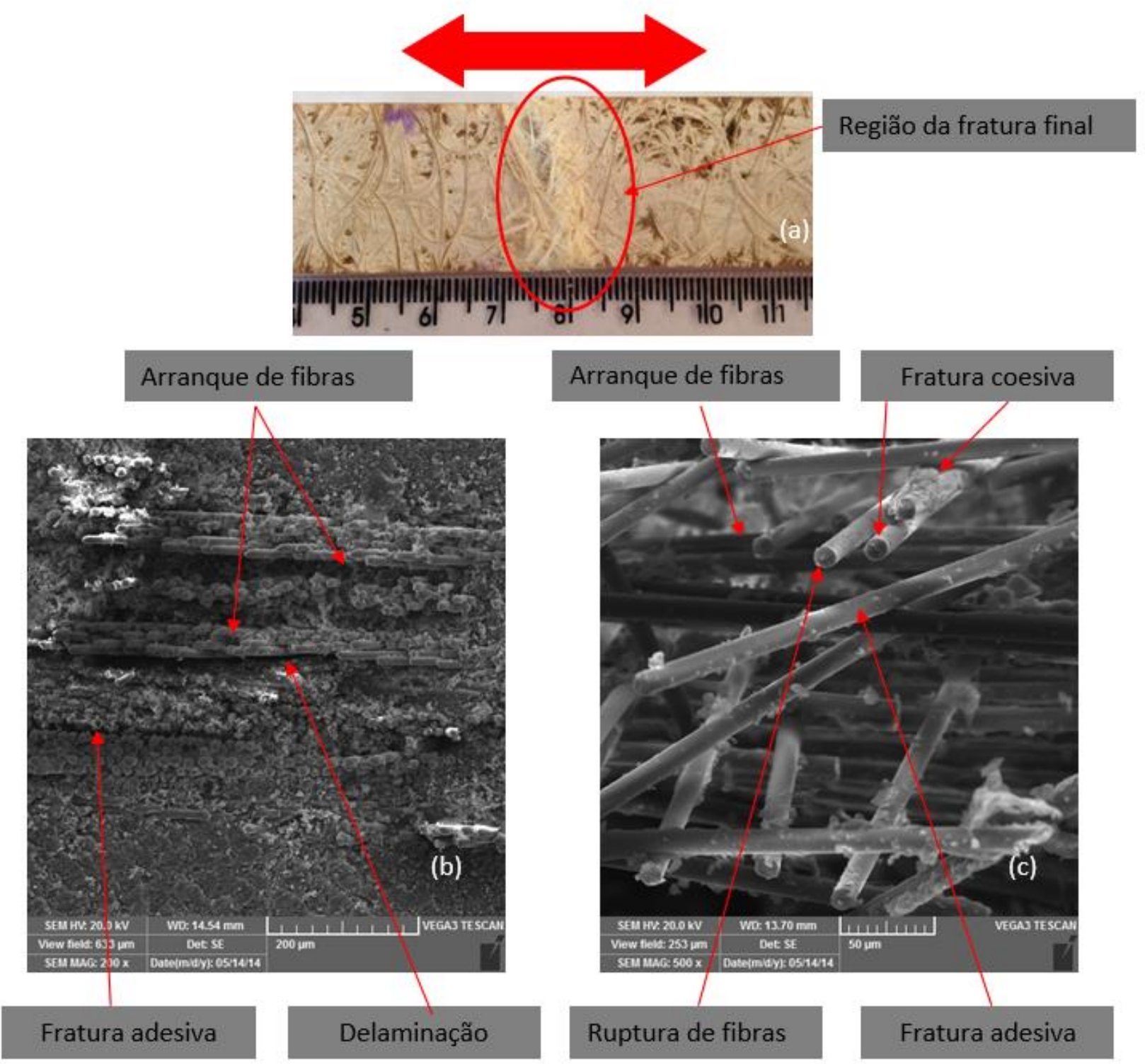

Figura 5: Região da fratura final do corpo de prova tracionado na após envelhecimento (imerso em água do mar).

$\mathrm{Na}$ figura 5(a) verifica-se que o corpo de prova está com toda a sua área embranquecida devido ao longo período de imersão em água e também é observada a presença de desaderência fibra/matriz sendo evidenciada pela presença de fibras de vidro expostas e também que o corpo de prova se rompeu de fato mediante o esforço de tração pela fratura está perpendicular ao esforço, esta imagem foi capturada com câmara digital. A figura 5(b e c) as imagens foram obtidas mediante MEV e observa-se na figura $5(\mathrm{~b})$ a presença de arranque de fibras, sendo constatado pela presença de furos; além disso é possível ver também a fratura adesiva fibra/matriz e a delaminação. Na figura 5(c) observam-se a presença de arranque de fibras, fratura adesiva na interface fibra/matriz, além disso a fratura coesiva na fibra, sendo confirmada pela característica da fratura angular na ponta da fibra e fratura coesiva na matriz, evidenciada pela presença de matriz na fibra. 
É possível observar que para ambas as condições de ensaio a fratura final dos corpos de prova tracionados, estado original e envelhecido, foi do tipo angular e no meio do galgo (LGM Lateral gage middle), conforme estabelece a norma ASTM D 3039-08.

\section{CONCLUSÕES}

Conforme os resultados acima apresentados, o polímero reforçado por fibra de vidro (PRFV), após a realização do ensaio de tração uniaxial, observou-se uma grande variação na resistência à tração, porém detectou-se uma pequena perda na rigidez quando o compósito foi imerso em água do mar; além disso referente a característica da fratura final independente do condicionamento ambos apresentaram fraturas semelhantes. Diante disso, apesar das perdas nas propriedades mecânicas após a exposição, este material pode ser bem utilizado nesta condição, uma vez uma vez sendo considerado estas perdas durante a fase de elaboração do projeto estrutural do componente mecânico.

\section{AGRADECIMENTOS}

Agradecemos a CNPq e ao IFRN pelo apoio financeiro na concessão de bolsas de iniciação científica.

\section{REFERÊNCIAS}

ASTM D 3039: Standard Test Method for Tensile Properties of Oriented Fiber Composites, 2008.

ASTM D 792: Standard Test Methods for Specific Gravity and Density of Plastics by Displacement - American Society for Testing and Materials, 2008.

ASTM D 3171: Standard Test Methods for Constituent Content of Composite Materials, 2004.

ASTM D 2563: Standard Practice for Classifying Visual Defects in Glass Reinforced Plastic Laminate Parts ${ }^{1}, 2008$.

Boger L., Wichmann M., Meyer, L. \& Schulte, K. (2008). Load and health monitoring in glass fiber reinforced composites with an electrically conductive nanocomposite epoxy matrix. Composite Science Technologies. 68 (7-8), 1886 - 1894.

Cavalcanti, W. S., Carvalho, L. H. \& Lima, A. G. B. (2005). Difusão de água em compósito poliéster insaturado reforçados por tecido híbrido de juta-vidro: modelagem e simulação. Revista Matéria. 10 (1), 14-23.

Felipe, Renata Carla Tavares Santos (2012). Envelhecimento ambiental acelerado em PRF a base de tecidos híbridos kevlar/vidro: propriedades e instabilidade estrutural. (Tese de doutorado) Universidade Federal do Rio Grande do Norte, Natal, RN.

Gupta, N., Brar, B.S. \& Woldesenbet E. (2001). Effect of filler addition on the compressive and impact properties of glass fibre reinforced epoxy. Bulletin of Materials Science. 24 (2), 219223.

Hull, D. \& Clyne, T. W. (1996). An introduction to composite materials Ed. Cambridge University Press, Cambridge.

Loyola, B. R., Saponara, V. L. \& Loh, K. J. (2010). In situ strain monitoring of fiber-reinforced polymers using embedded piezoresistive nanocomposites. Journal of Materials Science, 45 (24), 6786-6798. 
Leonard, L. W. H; Wong, K. J \& Low, K. O. J (2009). Fracture behaviour of glass fibre reinforced polyester composite. Journal of Materials: Design and Applications. 223 (2), 83-89.

Mendonça, P. T. R. (2005). Materiais compostos \& estruturas-sanduíches. Ed: Manoele.

Sathishkumar ,T.P., Satheeshkumar, S. \& Naveen, J. (2014). Glass fiber-reinforced polymer composites - a review. Journal of Reinforced Plastics and Composites. 33 (13), 1258-1275.

Rafiee, R. \& Reshadi, F. (2014). Simulation of functional failure in GRP mortar pipes. Composite Structures Elsevier. 113, 155-163.

Sridhar, I. \& Venkatesha, C. S. (2013). Variation of damping property of polymer composite under saline water treatment. International journal of innovations in engineering and technology. International Journal of Innovations in Engineering and Technology. 2 (1), 420-423.

Todo, M., Nakamura, T., Mada, T. \& Takahashi, K. (2000). Effects of moisture absorption on the dynamic interlaminar fracture toughness of carbon/epoxy composites. Journal of Composite Materials. 34 (8), 630 - 648.

Zhai, Z., Chen, X., He, Z., Ye, J. \& Zhu, X. (2014). Micromechanical modeling on the ratedependent viscoplastic behavior of polymer composites with thermal residual stress effect. Journal of Reinforced Plastics and Composites. 33 (17), 1574-1589. 